\title{
Convergences about the river. Transmedia Narrative for cultural identity
}

\author{
María Elena Tosello \\ Universidad Nacional del Litoral | Argentina | maritosello@gmail.com \\ Micaela Block \\ Universidad Nacional del Litoral | Argentina | micaela.block@gmail.com \\ Silvana Mariel Fontana \\ Universidad Nacional del Litoral | Argentina | silufontana@gmail.com \\ Patricia Mines \\ Universidad Nacional del Litoral | Argentina | patimines@gmail.com
}

\begin{abstract}
Visualizing the richness of a locality cultural heritage for its value as a source of social identity, requires means and actions that contribute to recognize it. The design of a documentary transmedia narrative that incorporates territorial navigation and integrates open and multiple citizen participation, constitutes an action aimed at disseminating and enhancing the city's immaterial cultural assets. The study made possible to survey the intangible heritage manifestations and to analyze the relevance of the available media and their communicative potential. It also constitutes an initial step for the creation of a useful database to manage its safeguard.
\end{abstract}

Keywords: Cultural identity; Intangible Cultural Heritage; Documentary Transmedia Narrative; Territorial navigation.

\section{INTRODUCCIÓN}

Cuando se vivencian profundos cambios, con constantes avances e impactos tecnológicos, la práctica de las disciplinas proyectuales no puede permanecer ajena ni indiferente. Así, en un contexto en el que el diseño desempeña un rol decisivo para el sostenimiento de la vida, el presente trabajo busca realizar un aporte original y creativo a partir de la integración de dos campos de conocimiento: el patrimonio cultural inmaterial $(\mathrm{PCl})$ y la narrativa transmedia documental (NTD).

Si bien a nivel mundial existen numerosos proyectos de investigación y salvaguardia del patrimonio cultural inmaterial, en Argentina las gestiones gubernamentales de relevamiento de estas manifestaciones patrimoniales son escasas, mientras que a nivel local son insipientes y desarticuladas. Por otro lado, el ámbito de la narrativa transmedia -en el que las tensiones se presentan entre ficción/no ficción- evidencia una limitada producción de proyectos transmedia documentales sobre patrimonio cultural inmaterial en general, y una producción casi nula en la ciudad de Santa Fe, capital de la provincia de Santa $\mathrm{Fe}$.

Frente a estas circunstancias, entendemos que visibilizar la riqueza patrimonial de una localidad para su reconocimiento como fuente de identidad social, requiere medios y acciones que contribuyan a reconocerla y valorarla, permitiendo definir el sentido de su conservación para toda la sociedad. Así, el diseño de una narrativa transmedia documental que incorpore la navegación territorial e integre la participación ciudadana abierta y múltiple, constituirá una acción orientada a la difusión y puesta en valor de los bienes culturales inmateriales de la ciudad y un paso inicial para la conformación de una base de datos útil para gestiones de salvaguardia.

Por este motivo, la investigación propuso abordar un distrito costero distintivo de la ciudad de Santa Fe -en el litoral argentino-, que integra una región caracterizada por la presencia de riachos, arroyos e islas que poseen alta biodiversidad, con un patrimonio natural y cultural asociado a esa riqueza y a las huellas de los usos y procesos productivos que en ellos tienen y tuvieron lugar (Mines, Tosello, Mihura, 2015). En base al desarrollo de experiencias de investigación y extensión universitaria (proyectos que vienen construyendo vínculos con la población local vulnerable a partir del reconocimiento del patrimonio -en sentido amplio- como recurso para vivir), y a informaciones, personajes y prácticas significativas así identificadas, el estudio permitió abordar manifestaciones patrimoniales inmateriales y analizar la pertinencia de los medios disponibles y sus potencialidades comunicativas, para diseñar un proyecto transmedia documental sobre dicho $\mathrm{PCl}$ que, a su vez, admita y posibilite la recepción de aportes de la propia comunidad. 


\section{METODOLOGÍA}

Para alcanzar los objetivos planteados, la investigación adoptó una metodología de tipo analítica-proyectual desde una perspectiva cualitativa. El trabajo se organizó en tres grandes etapas interrelacionadas: una primera de revisión bibliográfica para establecer los conceptos medulares que fundamentarán el proyecto; una segunda de análisis de casos análogos con el fin de definir premisas de diseño a considerar en la propuesta proyectual; y una tercera de desarrollo de un prototipo que busca alcanzar soluciones satisficientes (Simon, 2006).

\section{DEFINICIONES}

Respecto a los campos que atraviesan la investigación y considerando las múltiples acepciones de los conceptos patrimonio cultural y narrativa transmedia, en esta primera etapa fue necesario revisar la evolución de las definiciones y analizar sus versiones actuales.

Frente a la perspectiva que define el patrimonio como un binomio material-inmaterial que se presenta a través de las costumbres sociales en forma compleja y variada, adoptamos lo que la Organización de las Naciones Unidas para la Educación, la Ciencia y la Cultura, UNESCO (2003) establece como Patrimonio Cultural Inmaterial:

Los usos, representaciones, expresiones, conocimientos y técnicas -junto con los instrumentos, objetos, artefactos y espacios culturales que les son inherentes- que las comunidades, los grupos y en algunos casos los individuos reconozcan como parte integrante de su patrimonio cultural. Este patrimonio cultural inmaterial, que se transmite de generación en generación, es recreado constantemente por las comunidades y grupos en función de su entorno, su interacción con la naturaleza y su historia, infundiéndole un sentimiento de identidad y continuidad y contribuyendo así a promover el respeto de la diversidad cultural y la creatividad humana. (p. 2)

Asimismo, consideramos los ámbitos que incluye el Patrimonio Cutural Inmaterial: las tradiciones $y$ expresiones orales, comprendido el idioma como vehículo del PCl; las artes del espectáculo; los usos sociales, rituales y actos festivos; los conocimientos y usos relacionados con la naturaleza y el universo; y las técnicas artesanales tradicionales.

Respecto al PCl cabe destacar su fragilidad, que es mayor cuando se trata de contextos de gran vulnerabilidad social, con escasos recursos para su acumulación, expansión y perfeccionamiento (García Canclini, 1999), ya que esta característica -propia de la naturaleza y del modo de transmisión de este patrimonio- exige acciones de salvaguardia que incluyan alguna forma material -como pueden ser los archivos o inventarios- para su recolección y registro, y que también admitan la participación de los portadores y comunidades donde este patrimonio se circunscribe.

En cuanto a las narrativas, las industrias culturales asumieron formas transmediales de comunicación luego de los procesos de convergencia (Jenkins, 2008) que atravesó la ecología de medios, determinados por los profundos cambios que provocaron el desarrollo de las tecnologías digitales y las redes, particularmente de Internet, en los procesos de producción, circulación y consumo de contenidos.

En este sentido, la Narrativa Transmedia es "un tipo de relato que se despliega a través de múltiples medios y plataformas de comunicación, y en el cual una parte de los consumidores asume un rol activo en ese proceso de expansión" (Scolari, 2013: 46).

Además, la NT incluye ficción y no ficción. El macro género de no ficción abarca diversas formas de expresión, entre ellas el documental, un género que en su fase actual "presenta los elementos sustanciales de una narrativa a través de varios soportes y plataformas con el fin de propiciar una experiencia unificada y coordinada" (GifreuCastells, 2015, p. 1155).

Acerca de la producción, una Narrativas Transmedia Documental puede adoptar alguno de los cuatro modelos que establece Porto Renó (2014): estructurado, de visualización navegable, análogo-digital, o de navegación territorial. Entre estas opciones destacamos el modelo que promueve la navegación, involucrando a los participantes en ambientes no sólo virtuales, sino también físicos. Así, en esta propuesta, el espacio urbano se asume como plataforma narrativa transversal que permite vincular las historias con los espacios y el tiempo en que se experimentan (Irigaray, 2016).

\section{CASOS ANÁLOGOS}

La segunda etapa metodológica consistió en el análisis de tres NTD latinoamericanas: "Pregoneros de Medellín", "O som dos sinos" y "Tras los pasos del hombre bestia", que fueron seleccionadas por incorporar el modelo de navegación territorial; difundir y poner en valor el $\mathrm{PCl}$; datar de los últimos ocho años; y poseer premios o reconocimientos.

Con el objetivo de identificar las características generales y particulares de las unidades muestrales, y conocer las fortalezas y debilidades del diseño de estas narrativas para tenerlas en cuenta en el desarrollo del proyecto, se realizó un análisis detallado y ordenado del corpus seleccionado. Para ello, se definieron las categorías: Sintaxis, Tecnología e Interpretante, en correspondencia con las dimensiones del signo triádico de Peirce, resignificadas por Magariños de Morentin y Güerri: Forma, Existencia y Valor. Estas, a su vez, permitieron abarcar y organizar todos los componentes de una NTD que plantea Robert Pratten (2015): tema, historia, protagonistas, caracterización y relaciones, objetos significativos, ubicaciones, períodos y mensaje, conformaron la categoría Valor/Interpretante; diseño del mundo narrativo, modalidad de realismo, ejes de la experiencia, estilo, color y fuentes, presencia y tratamiento de signo identificador, integraron la categoría Forma/Sintaxis; modelo de producción, mapa expansivo, y contenido de medios y plataformas, constituyeron la categoría Existencia/Tecnología.

\section{DEFINICIÓN DE LA TEMÁTICA}

Para diseñar el proyecto, en la tercera y última etapa metodológica, fue necesario establecer las 
manifestaciones culturales que incluiría el documental transmedia. A tal fin, se elaboró un cuestionario guía y se realizaron siete entrevistas a profesores y personalidades destacadas de la cultura local, en relación al patrimonio inmaterial de la ciudad. A partir de estas entrevistas, se definió a los saberes insulares como temática del proyecto, contemplando la disponibilidad de material sobre dicho patrimonio; la oportunidad de nutrir el contenido a partir del contacto con residentes de la zona identificados como informantes claves; y la posibilidad de iniciar la construcción de una base de datos que integre otras manifestaciones patrimoniales de la región.

La definición de la temática fue decisiva para el diseño de la historia, del mundo narrativo y su estilo, del modelo de producción y del mapa expansivo.

\section{RESULTADOS}

El proceso teórico-analítico realizado derivó en el desarrollo de una propuesta a nivel de prototipo que fue evaluada por profesionales con distintos perfiles, y posteriormente ajustada. Así, se elaboró un proyecto de narrativa transmedia documental para la difusión y puesta en valor del PCl de la región ribereña, originado en la fuerte relación de los habitantes con el río, las lagunas e islas, con el objetivo de promover su reconocimiento social en tanto se trata de manifestaciones propias de la identidad cultural local.

\section{LA HISTORIA Y EL MUNDO NARRATIVO}

En esta historia, la fuente primaria de información son los propios protagonistas: hombres y mujeres que viven en las islas o en estrecha relación con ellas. En efecto, la narrativa propone la cultura local como eje de la experiencia: los relatos, objetos, personas y lugares reales constituyen la trama de la historia.

Así, relatos de baqueanos, historias de islas y conocimientos técnicos y populares, que se recopilaron en visitas al sitio, fueron las manifestaciones abordadas que se circunscriben a -por lo menos- dos ámbitos patrimoniales: el de las tradiciones y expresiones orales, y el de los conocimientos y usos relacionados con el universo. Además, al concebir el patrimonio como un binomio material-inmaterial, el proyecto consideró los signos tangibles inherentes a los distintos saberes y a sus portadores, como son: los instrumentos, objetos, artefactos y espacios que posibilitan las prácticas sociales y contribuyen al relato.

El estilo del mundo narrativo combina principalmente fotografías e ilustraciones. Debido al carácter testimonial que presenta, se recurre a la fotografía directa para mostrar el contexto geográfico insular, así como a los protagonistas y el desarrollo de sus prácticas. Las ilustraciones son dibujos lineales y se emplean en blanco -sobre fotografías o planos de color- para representar los elementos materiales y naturales relativos a los distintos saberes.

El título de la narrativa conforma el signo identificador (Fig. 1) que se aplica a todas las producciones del mundo narrativo.

\section{saberes $\approx$ INSULARES $\approx$}

Figura 1: Signo identificador de la NTD. Se diseña a partir de leves ondulaciones que recuperan el movimiento del río, uno de los elementos significativos de la historia.

Además, la paleta cromática de la narrativa incorpora dos de los colores presentes en el paisaje isleño: el marrón característico de las costas y del agua de río, y el celeste visible en el cielo, principalmente cerca del horizonte.

En cuanto al sonido, para contribuir al realismo de la historia, el estilo del mundo narrativo incorpora tres tipos de sonido: la voz humana (los diálogos que estructuran las escenas), los ruidos (el sonido ambiente) y una composición musical que recupera los sonidos de la naturaleza.

\section{MODELO DE PRODUCCIÓN Y MAPA EXPANSIVO}

En función al contenido, fue posible identificar distintos niveles de información, establecer la pertinencia y el orden de los medios a utilizar, así como definir el recorrido de los usuarios o prosumidores.

Se desarrolló la trama narrativa sincronizando diferentes medios y plataformas, según el modelo productivo de navegación territorial, con el objetivo de asumir el espacio urbano como plataforma narrativa transversal. La NTD quedó conformada por producciones digitales (redes sociales, página web y documental web interactivo), y físicas (audiovisuales urbanos, eventos de presentación, gráfica en buses, afiches callejeros y postales con Realidad Aumentada).

En el cronograma de medios (Fig. 2), se estipuló el orden de lanzamiento y la continuidad de las experiencias, con posibilidad de prolongar su desarrollo y expansión a partir del aporte de nuevos contenidos. De esta forma, el proyecto ofrece contenido interactivo (que se encuentra siempre a disposición del usuario), y también programado (su lanzamiento es definido en la línea de tiempo, independientemente de la demanda de la audiencia).

Se propuso comenzar con la creación de una página oficial en Facebook (obra seminal de la narrativa) y, a continuación, publicar el sitio web (obra núcleo que potencia el sistema). Luego, las proyecciones audiovisuales que estrenan el canal de YouTube y la cuenta de Instagram, el evento de presentación del proyecto y la distribución de afiches callejeros en la ciudad. A continuación, el lanzamiento de gráfica en buses y el estreno del documental web, seguido por la distribución de postales con RA.

Además, el proyecto planteó la realización de un largometraje documental sobre los distintos saberes insulares, a ser presentado en los cines locales y posteriormente distribuido en instituciones públicas (vecinales, bibliotecas y escuelas) de los barrios costeros, quedando a disposición de la propia comunidad. Cabe 


\begin{tabular}{|c|c|c|c|c|c|c|}
\hline Medio & & Plataforma & MES 1 & MES 2 & MES 3 & MES 4 \\
\hline saberes & Redes sociales & Digital & & & & \\
\hline & Sitio web & Digital & & & & \\
\hline & Audiovisuales urbanos & Física & & $\bullet-1$ & & \\
\hline & Evento de presentación & Física & & $\phi$ & & \\
\hline & Afiches callejeros & Física & & $\bullet$ & & \\
\hline & Gráfica en buses & Física & & & & \\
\hline & Documental web & Digital & & & & \\
\hline aristing & Postales con RA & Física & & $\phi$ & & \\
\hline
\end{tabular}

Figura 2: Cronograma y sincronización de medios de la NTD "Saberes Insulares".

aclarar que la fecha de realización no fue registrada en el cronograma ya que su producción podía incluir los aportes que la comunidad realizara.

Por su complejidad y dado que conforma la obra núcleo, se destaca el sitio web. En tanto meta-medio, esta plataforma posibilita integrar el contenido del proyecto en sus distintos formatos y concentrar la mayor cantidad de conexiones entre los medios de la narrativa. La información se organiza en seis secciones: Proyecto describe los objetivos, las producciones que conforman el universo narrativo y su equipo responsable, y reúne vínculos a investigaciones, notas periodísticas y diversos materiales sobre el patrimonio en cuestión; Patrimonio explica qué es el PCI según la UNESCO y cuáles son los ámbitos en que se desarrolla, a partir de ejemplos de manifestaciones locales; Conocimientos reúne, en una pantalla interactiva, testimonios y distintos saberes populares sobre los efectos de la luna en la flora y la fauna de las islas; Técnicas redirige al documental web que se aloja en un sitio independiente; Relatos presenta, en un mapa interactivo, historias de islas narradas por integrantes de la propia comunidad; y Participá ofrece un formulario a través del cual los usuarios pueden colaborar en el relevamiento de manifestaciones del patrimonio cultural inmaterial local (Fig. 3).
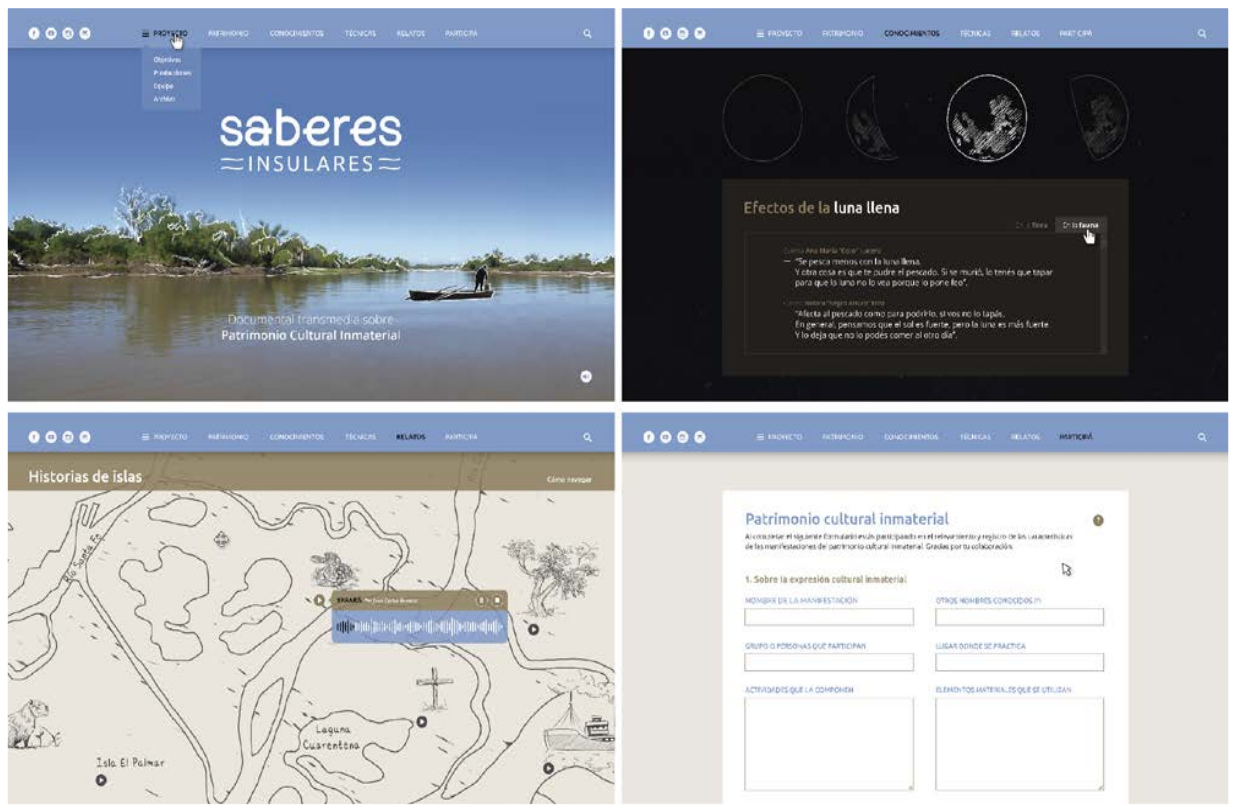

Figura 3: Sitio web de la NTD "Saberes Insulares". Páginas de "Inicio", "Proyecto", "Relatos" y "Participá". 
El audiovisual urbano expone contenido relacionado al saber popular con un doble objetivo: generar intriga en el espectador acerca de los efectos de la luna y difundir el proyecto a partir de la presencia de la marca, la dirección del sitio web y las redes sociales. Esta intervención adopta dos modalidades de visualización: el videomapping (en el centro de la ciudad, sobre cartelería y muros medianeros ubicados en arterias de alto tránsito y visibles a gran distancia), y la reproducción móvil (en un vehículo con pantalla led que recorre los distritos costeros).

La presentación oficial del proyecto consiste en un evento público que se realiza en un espacio con vista privilegiada a la zona de islas. Incluye la proyección de un audiovisual que resume el mundo narrativo y el obsequio de postales de RA a los asistentes.

Una serie de afiches se distribuye en las marquesinas municipales dispuestas a lo largo y ancho de la ciudad. Esta campaña en vía pública presenta distintas áreas de la región insular junto a un fragmento de la historia que da nombre a la isla referenciada. Además, invita a escanear un código QR para acceder a un material audiovisual alojado en YouTube- sobre la historia de los nombres con que los lugareños conocen a las islas.

En otra instancia, la gráfica en los buses urbanos promociona el documental web, particularmente en las líneas que recorren tanto el distrito de la costa como la zona portuaria de la ciudad.

El documental web interactivo refiere a uno de los saberes insulares. Se estructura en dos secciones principales: Portadores, contiene producciones audiovisuales sobre la actividad que cada protagonista desarrolla, y Recursos, visualiza una galería interactiva de los elementos materiales más significativos para las técnicas. Además, este webdoc vincula al sitio web del proyecto y a Facebook, donde se puede encontrar más información sobre los portadores del saber y sus técnicas.

Las tarjetas postales con realidad aumentada consisten en una serie de piezas que ofrecen relatos insulares, gráficos y audiovisuales. Las tarjetas promueven la participación de un público específico e invitan a difundir el proyecto, así como a completar el dorso de la postal con alguna historia de islas que conozcan.

Por último, cabe destacar que la propuesta incluye Facebook, Instagram y YouTube. Las tres redes sociales se complementan y colaboran en la expansión del relato, dando a conocer las diferentes instancias del proyecto e incitando a la participación.

Todas las producciones del universo transmedia se conectan con el sitio web -la obra núcleo- y las redes sociales. De este modo, las múltiples interconexiones que

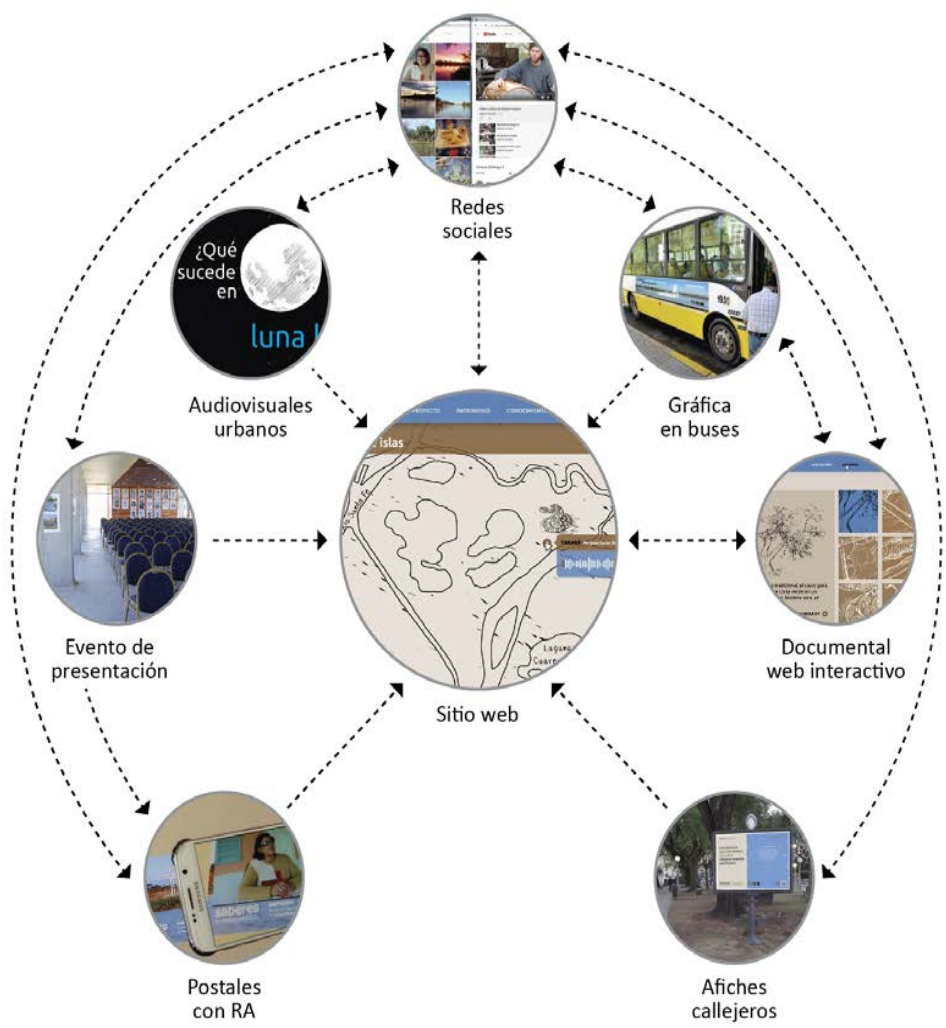

Figura 4: Recorrido del usuario que habilita el universo transmedia "Saberes Insulares". 
presenta el mundo narrativo permiten al usuario comenzar el recorrido por cualquier producción y saltar entre las distintas plataformas, con la libertad de elegir los medios y la extensión de la propia experiencia (Fig. 4).

\section{CONCLUSIONES}

La difusión y puesta en valor del Patrimonio Cultural Inmaterial resulta un tema de interés para la ciudad y la región debido a su valor como fuente de identidad cultural local.

A partir del estudio, que fusiona los sistemas físicos, biológicos y digitales, se pudo observar que el modelo de navegación territorial permite alcanzar a un público amplio, al tiempo que otorga prioridad al local. La estrategia apuntó a una población en particular (la del distrito costero), que convive diariamente con el $\mathrm{PCl}$, y a la población en general (de la ciudad), que en su mayoría desconoce este patrimonio.

La complementariedad entre los medios -donde las plataformas digitales no encuentran fronteras y las físicas asignan sentido de exclusividad al contenido- fue necesaria para llegar a una audiencia diversa y aumentar las posibilidades de que la comunidad realice aportes. Una participación amplia, sumada al conocimiento local, resulta fundamental en el proceso de identificación y relevamiento de manifestaciones patrimoniales. Asimismo, el modelo de navegación territorial es propicio para complementar tareas que desarrollan los especialistas en salvaguardia del $\mathrm{PCl}$, constituyendo un aporte original de los medios.

La investigación permitió comprobar que el tema es pertinente para su desarrollo en el campo del Diseño de la Comunicación Visual, integrado al Diseño Transmedia. Así, es pertinente la participación de comunicadores visuales en el desarrollo de estrategias de puesta en valor del $\mathrm{PCl}$, en tanto agentes culturales dentro de un equipo interdisciplinario.

Consideramos además que, a futuro, el proyecto podría fortalecerse a partir de la articulación en red con iniciativas en curso a escala del territorio completo de la micro región insular, en donde la búsqueda de consenso sobre saberes y actores, la articulación de intereses para cuidar estos frágiles territorios, y el diseño y la planificación de productos orientados al turismo cultural, serían los objetivos primordiales (Mines, Tosello, Mihura, Óp. Cit.). La narrativa requerirá entonces, adecuar su diseño al contexto en que esta se inscribe, ya que la elección de medios se encuentra supeditada no sólo al tema que se aborda, sino también al público destinatario. "Son las propias historias las que revelan cuál es la mejor plataforma o el mejor canal para acercarlas a la comunidad" (Lovato, 2015, p. 50).

\section{AGRADECIMIENTOS}

Damos las gracias a la Universidad Nacional del Litoral por el subsidio de la participación en este congreso.

\section{REFERENCIAS}

García Canclini, N. (1999). Los usos sociales del patrimonio cultural. En Aguilar Criado, E. (Coord.) Patrimonio Etnológico. Nuevas perspectivas de estudio (pp. 16-33). Andalucia: Instituto Andaluz de Patrimonio Histórico.

Gifreu-Castells, A. (2015). El uso del documental transmedia como herramienta para el cambio social. Análisis de casos focalizados en la desigualdad de género en el siglo XXI. En Nos Aldás, E., Arévalo Salinas, A. I. y Farnés, A. (Eds.), \#comunicambio: Comunicación y Sociedad Civil para el cambio social (pp. 1154-1177). Madrid: Fragua.

Güerri, C., Acebal, M., Alisio, J., Betancourt Ruiz, M., Binnevies, A. M., Bohórquez Nates, M., Pertot, W., Sastre, R., Voto, C.; (2016). Nonágono semiótico: un modelo operativo para la investigación cualitativa. Buenos Aires, Argentina: Eudeba; Ediciones UNL.

Irigaray, F. (2016). Documental Transmedia: narrativas espaciales y relatos expandidos. En Irigaray, F. y Renó, D. (Comps.), Transmediaciones. Creatividad, innovación y estrategias en nuevas narrativas (pp. 39-54). Buenos Aires: La Crujía.

Lovato, A. (2015). Del periodismo multimedia al periodismo transmedia Guiones para pensar nuevas narrativas. En Irigaray, F. y Lovato, A. (Eds.), Producciones transmedia de no ficción. Análisis, experiencias y tecnologías. (pp. 33-52). Rosario: UNR Editora. https://www.academia.edu/22718076/

Organización de las Naciones Unidas para la Educación, la Ciencia y la Cultura (2003). Convención para la Salvaguardia del Patrimonio Cultural Inmaterial. París: Autor.

Organización de las Naciones Unidas para la Educación, la Ciencia y la Cultura (2016). Textos fundamentales de la Convención para la Salvaguardia del Patrimonio Cultural Inmaterial de 2003. París: Autor.

Mines, P., Tosello, M. E., Mihura, E. (2015). Paisajes vulnerables: estrategias y espacios de diálogo para un turismo sustentable. XXXIV Encuentro Arquisur 2015 y XIX Congreso de Escuelas y Facultades Públicas de Arquitectura de los países de América del Sur (La Plata, Argentina). Disponible en: http://sedici.unlp.edu.ar/handle/10915/51801

Porto Renó, D. (2014). Formatos y técnicas para la producción de documentales transmedia. En Irigaray, F. y Lovato, A. (Eds.), Hacia una comunicación transmedia (pp.133-146). Rosario: UNR Editora.

Pratten, R. (2015). Getting Started with Transmedia Storytelling. A Practical Guide for Beginners. https://goo.gl/vxgTX1

Scolari, C. (2013). Narrativas transmedia. Cuando todos los medios cuentan. Barcelona: Planeta.

Simon, H. (2006). Las ciencias de lo artificial. Granada: Comares. 